\title{
The University of Waikato Usability Laboratory
}

\author{
Kirsten Thomson and Mark Apperley \\ Department of Computer Science \\ University of Waikato, Hamilton, New Zealand \\ kthomson@cs.waikato.ac.nz \\ m.apperley@cs.waikato.ac.nz
}

\begin{abstract}
The University of Waikato Usability Laboratory is an environment where researchers are able to study and assess the usability of products while being used by their intended users. It allows for flexible configuration, and in particular can accommodate studies involving groups of collaborating users. This paper describes the Usability Laboratory with a particular emphasis on its background-why the Laboratory was established - and the facilities and services that it provides.
\end{abstract}

\section{Introduction}

The University of Waikato Usability Laboratory was formally established in February 2001 by the HumanComputer Interaction ( $\mathrm{HCI})$ research group. A number of usability studies had been carried out during the previous four years, particularly in relation to the Computer Supported Collaborative Work project [6], using ad hoc facilities. This work had identified the need for a more formal and specialised environment to support and premote this type of activity.

One of the principal requirements of the $\mathrm{HCI}$ research group was for flexibility. We needed to be able to accommodate a wide variety of study types; from new widget evaluations through to workplace studies; and from those involving just single users through to studies of groups of collaborating users, either in a common space or at multiple locations. Although the requirement for single user studies is common, because much of our research relates to collaborative work [1,2], meeting this latter requirement was essential. It was also evident that typical pre-packaged usability laboratory systems [7] were unlikely to satisfy our needs. These requirements for flexibility, and the need to accommodate group user studies, implied a laboratory with at least two rooms in which user studies could take place, with at least one of these large enough for users working as a group within the same room. To meet these requirements we have developed a laboratory incorporating a large group-user room in addition to a traditional single-user room, both of which can be viewed from a common observation room. In providing facilities for studies involving the simultaneous use of these two rooms, it was also important that when only a single room was required, the laboratory could facilitate two studies in parallel, each utilising one of the two available spaces.

To support this flexible use of the laboratory space, it became an important requirement also to provide flexibility in the arrangement of video and audio equipment used for recording the studies. All of the video equipment can be configured and operated under computer control from the observation room, allowing rapid reconfiguration of the space from, for example, a single study involving both user rooms to two independent studies

In this paper we outline the principal goals we set out to achieve (Section 2) and then describe in detail the facilities we have constructed to meet these goals (Section 3). Section 4 outlines the services provided by the laboratory, and in Section 5 we provide a brief overview of some of the studies carried out in the laboratory so far.

\section{Goals}

In establishing a usability laboratory to meet the needs of our own research (see Section 1), we have also made a predominant focus the promotion and application of usability practices in IT development. The failure of many computer-based systems to be effective and efficient in meeting users' requirements has motivated our desire to promote usability engineering principles. We are promoting and facilitating the use of usability practices within the New Zealand IT industry through the provision of usability testing facilities, resources and services, and by educating IT developers to make them aware of the very important place of users and usability in the software development lifecycle. 
In order to achieve these goals, the laboratory has been established with three broad categories of client in mind:

(i) Researchers within our own HCI research group. Through our own experience we are able to ensure that the laboratory remains up-to-date, is well equipped, and can meet the needs of a wide range of usability studies.

(ii) Commercial users - the New Zealand IT industry. By encouraging and accommodating commercial clients, we are not only promoting usability practices in New Zealand industry, but we are able to provide them with support and services, and access to facilities not previously available.

(iii) Other researchers in New Zealand and overseas. Given the pre-eminent position of our laboratory in New Zealand, we are actively encouraging its use by colleagues within our own department, elsewhere in the University, and from other universities and research establishments.

We have established a comprehensive usability testing facility, such as not previously available in New Zealand. Our commitment to and experience in the usability field enables us to offer a range of usability services to these client groups, including the analysis, design and testing of IT systems.

\section{Facilities}

The Usability Laboratory comprises five rooms, two of which are testing rooms (one a single-user room, the other a group-user room), and another specifically equipped for observation. Of the two remaining rooms, one is a reception and the other an overview/planning room. Figure 1 provides a plan of the space and shows the functions of each of these rooms.

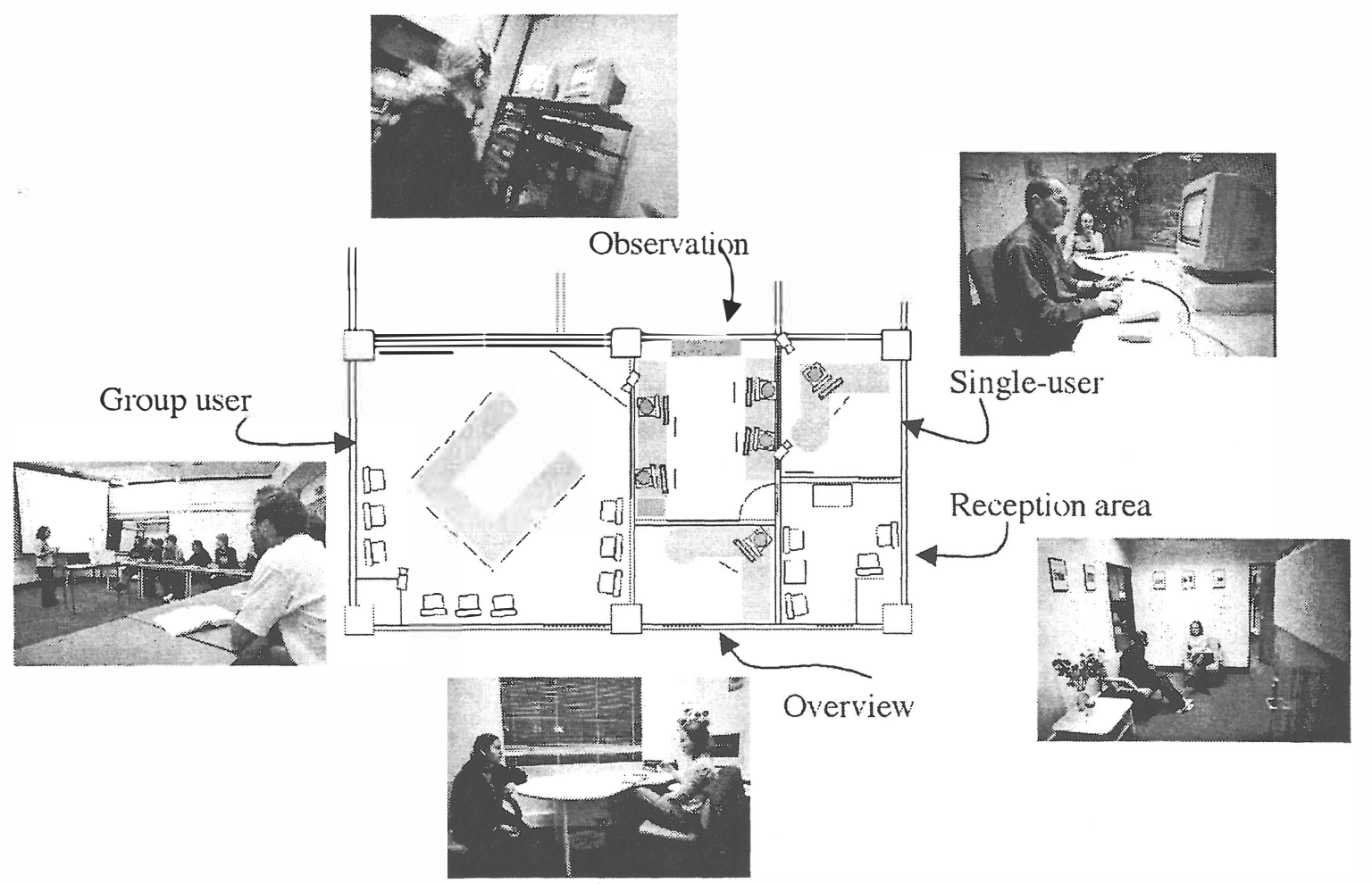

Figure 1. The layout and functionality of the Usability Laboratory

\subsection{User Testing Rooms}

The Laboratory provides two separate user testing rooms, each with its own purpose. The first is a single-user room, intended for studies involving one or two users at a time. The room contains a single desktop computer (with connected scan converter) and facilities to add additional computers as required. The second of the user testing rooms is a group-user room. This room is large (seven by seven metres) and can easily accommodate up to twenty people at a time, in a meeting or a classroom configuration, or arranged to emulate a workplace. A variety of studies may be conducted in the group-user room: from single-user to group interaction or multi-user studies. The furniture in the room is portable and customisable. The room also contains a large whiteboard, and 
two projector screens. Additional facilities consist of a portable printable whiteboard, and a portable large interactive display surface (LIDS). The group-user room also doubles as a departmental seminar room when not in use by the laboratory.

The Laboratory is equipped with four pan-tilt-zoom video conferencing cameras which can be moved to almost any position in either of the two rooms. They are relatively small and unobtrusive, and whilst normally attached to a dado running around the wall, they can be extended into the room on specially designed telescopic arms. Both user rooms are equipped with discrete ceiling mounted microphones, and have intercom connection from the observation room.

\subsection{Observation Room}

The observation room provides large one-way windows for direct viewing into each of the testing rooms, and a wide range of tools for recording and reviewing test proceedings. The video equipment is predominantly digital, and has facility for computer control. This equipment includes two four-quadrant video multiplexers, one of fixed configuration, and the other fully programmable, and a further picture-in-picture (two input) video multiplexer. The images from the multiplexers, the scan converter and/or the cameras, can be recorded onto two digital editing video recorders and viewed on any combination of the six 14 " and one 21 " monitors. Connections between cameras, multiplexers, recorders and monitors are controlled by two $8 \times 8$ video cross-bar-switches, ensuring that no reconnecting of cables is necessary to modify the laboratory configuration. A typical experimental composite video image is shown in Figure 2, where the user's screen (top left) has been captured using the scan converter, together with camera views of their face and the keyboard, on a single video tape, facilitating later analysis of their actions. The cameras, video recorders, multiplexers and cross-bar switches are all under computer control through RS232 serial connections. Software is being developed to provide total configuration and operational control from the two observers' computers, which are strategically placed beside the viewing windows into each of the user rooms.

Audio captured from the two user rooms can be accessed from multiple headsets in the viewing room, and can be recorded with the video signals as required. Intercom facilities are provided to allow the experimenter/observer to communicate with participants. Like the video, the audio facilities can be configured for a two-room single experiment, or two independent simultaneous experiments.

Up to six observers can be accommodated in the observation room at one time, but in addition to the one-way windows for direct viewing into each testing room, both the waiting area and the group-user room provide facilities from which additional observers can view the proceedings.

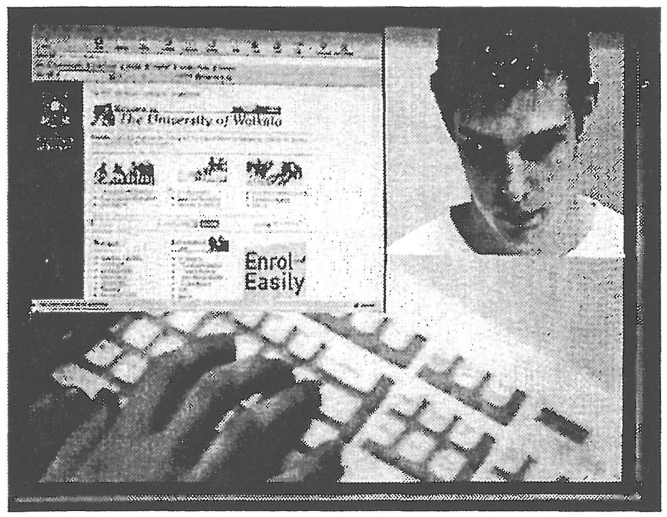

Figure 2. An example composite image captured on a single video channel

\subsection{Other Facilities}

The laboratory includes a reception room, a very important facility in conducting experiments. This provides a comfortable neutral location for waiting users, or alternatively space for discussion and planning, and can also be utilised as an additional viewing area for observers. The overview room allows for the planning and coordination of user studies, and provides a view, through a one-way window, of the proceedings in the observation room. 
Overall, the facilities provided by the laboratory are comprehensive. The ability to accommodate group studies, and the flexibility of the configuration, make this a unique laboratory for New Zealand, and one at the forefront internationally.

\section{Services}

In conjunction with these facilities, the Usability Laboratory also provides a range of services that utilise the experience and knowledge of those staff and students involved with the laboratory. The services range from the usability testing of paper-and-pencil prototypes to the evaluation of full working systems to identify usability issues, problems and improvement opportunities. This process may involve determining usability goals; creating test scenarios; providing supporting materials (instructions, questionnaires, training materials, etc.); testing facilities (as described in Section 3); recruiting users/subjects; collecting, analysing and interpreting quantitative and qualitative data; and drawing conclusions and making recommendations based on the test results.

Other services offered by the laboratory include user profiling, task analysis, the development of style guides and principles, interface design consulting and heuristic evaluation.

\section{Activities}

Since February 2001, when the Laboratory formally opened, a range of studies have been carried using the facilities. These include one project for an external client, two research-oriented projects within the department and two student projects forming part of an honours/graduate paper. Of the two research-oriented projects, the first was principally a comparison study of LIDS and desktop environments when used to organise files into separate folders (or categories) [3]. The goal was to measure the effectiveness of each environment to support group work and collaboration and to determine the advantages and disadvantages of using LIDS in this situation. The study also provided an opportunity to assess some of the ergonomic and interactive features of LIDS. The second research project was an evaluation of the installation and collection building processes of the New Zealand Digital Library (NZDL) [8]. The study was conducted in two parts; a comparison of the Greenstone Digital Library software command line interface and the Collector GUI approach for collection building, and an assessment of the processes involved with installing the Greenstone Digital Library software from CD-ROM [4].

The two teaching-related studies are (i) a holistic evaluation of the University of Waikato web site, conducted by five groups of four to six students, and (ii) a study conducted by a fifth-year student involving an exploratory evaluation of The Developer, a tool developed in-house to enable non-developers to easily add content to a web site [5].

Experience with these studies has shown the utility of the laboratory, and the importance of its highly flexible configuration.

\section{Summary}

This paper has described the recently established University of Waikato Usability Laboratory. The laboratory, set up by the Human-Computer Interaction research group, under the direction of Mark Apperley and with Kirsten Thomson as manager, provides a uniquely flexible configuration and can accommodate collaborative group studies. In addition to its research support goals, the laboratory has a predominant focus on the promotion and application of sound usability practices in the IT industry in New Zealand.

\section{Acknowledgements}

The HCI research group is supported by the Foundation for Research Science and Technology under the New Economy Research Fund contracts UOWX9910 and UOWX0011. 


\section{References}

[1]. Apperley, M., Fletcher, D., Rogers, W. and Thomson, K. (2000): Interactive Visualisation of a Travel Itinerary. In Proceedings of the Working Conference on Advanced Visual Interfaces, AVI '2000. Palermo, Italy May 23-26. ACM Press, New York. 221-226.

[2]. Apperley, M., and Masoodian, M. (2000): Supporting Collaboration and Engagement using a Whiteboardlike Display. CSCW2000 Workshop on Shared Environments to Support Face-to-Face Collaboration, 2226.

[3]. Boardman, R. and Thomson, K. (2001): Submitted to Interact '01 Conference Proceedings.

[4]. Nichols, D.M., Thomson, K. and Yeates, S. (2001): Usability and open-source software development. Elsewhere in these Proceedings.

[5]. Steel, A. and Thomson, K. (2001) The Developer: A development and support tool for content-based web sites. Elsewhere in these Proceedings.

[6]. Thomson, K, and Apperley, M.D. (1999): Developing a Graphical Timeline Representation for a Travel Itinerary. Working Paper 99/15. Department of Computer Science, University of Waikato.

[7]. Usability Systems, Inc (2001): http://www.usabilitylabs.com/

[8]. Witten, I.H., McNab, R., Jones, S., Cunningham, S.J., Bainbridge, D. and Apperley, M. (1999): Managing complexity in a distributed digital library. IEEE Computer, 32(2), 74-79. 